\title{
Neue Arzneimittel in der Onkologie: Merkmale klinischer Zulassungsstudien und Argumente für die rasche Durchführung unabhängiger klinischer Studien nach der Zulassung
}

\author{
Wolf-Dieter Ludwig ${ }^{\mathrm{a}, \mathrm{b}} \quad$ Gisela Schott ${ }^{\mathrm{b}}$ \\ ${ }^{a}$ Arzneimittelkommission der deutschen Ärzteschaft (AkdÄ), Berlin, \\ ${ }^{\mathrm{b}}$ Klinik für Hämatologie, Onkologie und Tumorimmunologie, HELIOS Klinikum Berlin-Buch, Deutschland
}

Schlüsselwörter

Neue onkologische Wirkstoffe - Klinische Studien .

Orphan Drugs · Zulassung · Versorgungsqualität

\section{Zusammenfassung}

Die Zulassung bzw. Indikationserweiterung für alle onkologischen Arzneimittel erfolgt heute in Europa basierend auf der Verordnung (EG) Nr. 726/2004 in einem zentralisierten Verfahren der European Medicines Agency (EMA). Untersuchungen der letzten Jahre haben Mängel in den Zulassungsstudien aufgezeigt. So werden beispielsweise die Vorgaben der EMA nicht immer konsequent beachtet und Studien werden nach Zwischenanalysen, die zu diesem Zeitpunkt eine bessere Wirksamkeit gegenüber dem Vergleichsarm zeigen, vorzeitig abgebrochen. Unsere aktuelle Auswertung der Europäischen Bewertungsberichte (Auswertungszeitraum: 01.01.2009 bis 13.08.2012) zu 29 Wirkstoffen für 39 onkologische Indikationen ergibt, dass sich die Qualität der Zulassungsstudien in verschiedener Hinsicht verbessert hat. So wurden meist die von der EMA und der Food and Drug Administration (FDA) empfohlenen primären Endpunkte - Gesamtüberleben und progressionsfreies Überleben - verwendet und nur eine Studie wurde als Phase-II-Studie ohne Vergleichsarm durchgeführt. Demgegenüber werden die onkologischen Arzneimittel zur Behandlung seltener Leiden (Orphan Drugs) auf der Basis von kleinen Studien zugelassen, die vielfach offen und nicht randomisiert durchgeführt werden und Surrogatendpunkte untersuchen. Nach der Zulassung ist für die Beantwortung der noch offenen patientenrelevanten Fragen die Durchführung von unabhängigen klinischen Studien erforderlich, für die verstärkt öffentliche Gelder bereitgestellt und bürokratische Hürden abgebaut werden müssen. Nur so kann ein effizienter Umgang mit begrenzten Gesundheitsressourcen ermöglicht und die Versorgungsqualität von Tumorpatienten verbessert werden.
Keywords

New oncology drugs - Clinical trials .

Orphan drugs · Approval - Quality of health care

\section{Summary}

New Drugs in Oncology - Features of Clinical Trials for Market Authorisation and Arguments for the Rapid Implementation of Independent Clinical Trials Following Approval

The market authorisation or extension of indication for all oncology drugs in Europe is now based on Regulation (EC) No. 726/2004, a centralised procedure of the European Medicines Agency (EMA). Studies in recent years have highlighted deficiencies in pivotal studies. For example, the requirements of the EMA are not always consistently followed and studies are stopped prematurely after only interim analysis that at this time point shows improved efficacy with regard to the comparator arm. Our current analysis of the European Assessment Reports (reporting period: 01/01/2009 to $08 / 13 / 2012$ ) on 29 drugs for 39 oncology indications shows that the quality of the trials for market authorisation has improved in several respects. Primary endpoints recommended by the EMA and the Food and Drug Administration (FDA) such as overall survival and progression-free survival are used, and only one study was conducted as a phase II trial with no comparator arm. In contrast, oncology drugs that are approved for the treatment of rare diseases (orphan drugs) are based on small studies which are often carried out without blinding, are not randomised and investigate surrogate endpoints. To answer patient-relevant issues following market authorisation, it is necessary to conduct independent clinical studies. Increased public funding needs to be provided and bureaucratic hurdles have to be reduced. Only this will permit a more efficient use of limited health care resources and allow to improve the quality of care for cancer patients.

\section{KARGER \\ Fax +497614520714 \\ Information@Karger.com}

www.karger.com (c) 2013 S. Karger GmbH, Freiburg

0378-584X/13/3614-0017\$38.00/0

Accessible online at:

www.karger.com/onk
Prof. Dr. Wolf-Dieter Ludwig

Arzneimittelkommission der deutschen Ärzteschaf

Herbert-Lewin-Platz 1, 10623 Berlin, Deutschland

wolf-dieter.ludwig@akdae.de 
Neue onkologische Arzneimittel: Status quo der Zulassungsstudien

Im Jahr 2011 wurden in den USA 30 und in Europa 22 neue Wirkstoffe zugelassen, darunter 8 bzw. 7 für die Behandlung onkologischer Indikationen [1]. Der bereits über mehrere Jahre zu beobachtende Trend hält somit an: Fast ein Drittel der neu zugelassenen Arzneimittel sind für die Behandlung solider Tumore bzw. hämatologischer Neoplasien vorgesehen - häufig nach beschleunigten Zulassungsverfahren und/ oder als Arzneimittel für die Behandlung von seltenen Leiden. Neben der demografischen Entwicklung und dem Auslaufen der Patente für Blockbuster zur Behandlung der Volkskrankheiten sind hierfür insbesondere verantwortlich: Fortschritte in der molekularbiologischen Charakterisierung des Tumorwachstums und daraus resultierend die Entwicklung einer Vielzahl neuer «zielgerichteter» Wirkstoffe, der medizinische Bedarf an besser wirksamen medikamentösen Therapieoptionen bei fortgeschrittenen Tumorerkrankungen sowie der sehr lukrative Markt für onkologische Arzneimittel.

Die Zulassung bzw. Indikationserweiterung für alle onkologischen Arzneimittel erfolgt heute in Europa basierend auf der Verordnung (EG) Nr. 726/2004 in einem zentralisierten Verfahren der European Medicines Agency (EMA). Sie erfordert klinische Studien der Phase II bzw. III, für deren Durchführung regulatorische Vorgaben von der EMA detailliert beschrieben werden [2]. Sowohl für die explorativen Phase-I/II-Studien als auch für konfirmatorische Phase-IIIStudien werden in dieser Verordnung Empfehlungen ausgesprochen, unter anderem zur Charakterisierung des Patientenkollektivs und der Tumorerkrankung, zur Art der klinischen Studie und deren Design, zu den Ein- und Ausschlusskriterien und $\mathrm{zu}$ den klinisch relevanten Endpunkten der Studie [2]. Zulassungsstudien werden heute fast ausschließlich von pharmazeutischen Unternehmen gesponsert, konzipiert, durchgeführt sowie ausgewertet - mit dem Ziel, die Marktzulassung für ein neues Arzneimittel oder eine Ausweitung der Anwendungsgebiete zu erhalten.

Untersuchungen der letzten Jahre in Nordamerika und Europa haben Defizite in klinischen Zulassungsstudien für onkologische Arzneimittel aufgezeigt, die vor allem folgende Aspekte betreffen: das Studiendesign, die gewählten (Surrogat-)Endpunkte, die meistens (zu) restriktiv definierten Einund Ausschlusskriterien für Patienten und die kurzen Beobachtungs- bzw. Nachbeobachtungszeiträume [3]. Verantwortlich hierfür sind in erster Linie das kommerzielle Interesse der pharmazeutischen Unternehmen an rascher Markteinführung ihrer neuen, in der Regel sehr teuren Wirkstoffe, aber auch die nicht immer eindeutig formulierten oder konsequent beachteten regulatorischen Vorgaben.

Die Ergebnisse klinischer Zulassungsstudien hinsichtlich Wirksamkeit und Sicherheit neuer Wirkstoffe werden von der EMA und der Food and Drug Administration (FDA) häufig unterschiedlich bewertet. Dies veranschaulicht eindrücklich auch ein kürzlich publizierter Vergleich der durch die EMA bzw. FDA erteilten Zulassungen von neuen onkologischen Arzneimitteln [4]. Untersucht wurden 42 Arzneimittel in 100 Indikationen (hämatologische Neoplasien und solide Tumore), die zwischen 1995 und 2008 von der EMA zugelassen wurden. In 47 der 100 Indikationen bestanden Unterschiede zu den Zulassungen der FDA, wobei der jeweilige Wirkstoff in 19 der 47 Indikationen nur von einer der beiden regulatorischen Behörden zugelassen wurde. In weiteren 28 Indikationen bestanden Unterschiede in der Beschreibung der zugelassenen Indikation(en). Formulierungen unterschieden sich beispielsweise hinsichtlich der Patientenuntergruppe, für die das Arzneimittel zugelassen wurde, und der vorausgegangenen medikamentösen Therapie. Aufgrund der zum Zeitpunkt der Zulassung häufig unvollständigen Ergebnisse bezüglich Wirksamkeit und Sicherheit orientieren sich EMA und FDA bei der Abfassung der therapeutischen Indikationen an den Merkmalen der in den Zulassungsstudien behandelten Patienten. Die Übertragbarkeit dieser Ergebnisse auf den Versorgungsalltag (externe Validität) ist deshalb begrenzt und Zulassungen, die eine Durchführung weiterer klinischer Studien nach $\mathrm{Zu}$ lassung verbindlich vorschreiben, haben deutlich zugenommen [4]. Die in dieser Auswertung europäischer Experten aufgezeigten Unterschiede in den Entscheidungen der $\mathrm{Zu}$ lassungsbehörden sind klinisch relevant, da sie den Zugang der Patienten zu therapeutischen Optionen möglicherweise einschränken oder aber dazu führen, dass Patienten neue Arzneimittel erhalten, deren Nutzen-Risiko-Profil anderswo als negativ beurteilt wurde [4]. Da die Gründe für negative Beurteilungen eines Arzneimittels gewöhnlich nicht detailliert veröffentlicht werden, sind die Entscheidungen für die Onkologen in der Regel nicht nachvollziehbar und werden von wissenschaftlichen Fachgesellschaften auch häufig kritisiert [4]. Auch bei Versagen der Zulassung bzw. Widerruf einer Indikation bei onkologischen Wirkstoffen unterscheiden sich mitunter die Entscheidungen der EMA und der FDA, wie auch ein aktuelles Beispiel belegt: Rücknahme der Indikation «metastasiertes Mammakarzinom» für Bevacizumab in den USA [4]. Ein weiterer kritikwürdiger Aspekt betrifft die deutliche Zunahme von Zulassungen onkologischer Wirkstoffe im Rahmen von beschleunigten Zulassungsverfahren (FDA: accelerated approval (beschleunigte Zulassung); EMA: conditional marketing authorisation). Die Umwandlung derartiger Zulassungen - häufig basierend auf Ergebnissen zu Surrogatendpunkten - in eine reguläre Zulassung erfordert weitere, aussagekräftige klinische Studien nach Zulassung. Ergebnisse dieser Studien liegen in der Regel erst etwa 4 Jahre nach Zulassung vor und die Umwandlung der beschleunigten in eine reguläre Zulassung erfolgt insgesamt zu selten [5]. 
Tab. 1. Charakteristika von Studien für die Zulassung von Arzneimitteln zur Behandlung maligner Erkrankungen (EPAR erschienen zwischen 01.01.2009 und 13.08.2012)

\begin{tabular}{lc}
\hline & $\mathrm{n}(\%)$ \\
\hline Zulassungen/Zulassungserweiterungen & $39(100)$ \\
Neue Wirkstoffe & $15(38)$ \\
Indikationserweiterungen & $24(62)$ \\
Studien pro Zulassung/Zulassungserweiterung & $1-3$ \\
Nur 1 Zulassungsstudie & $35(90)$ \\
$>1$ Zulassungsstudie & $4(10)$ \\
Zulassungsstudien insgesamt & $44(100)$ \\
Studiendesign & \\
Offene RCT & $26(59)$ \\
Doppelt verblindete RCT & $17(39)$ \\
Offene einarmige Studie & $1(2)$ \\
Art des Endpunkts & \\
Progressionsfreies Überleben & $22(50)$ \\
Gesamtüberleben & $10(23)$ \\
Erkrankungsfreies Überleben & $4(9)$ \\
Progressionsfreies Überleben und Gesamtüberleben & $2(5)$ \\
Rezidivfreies Überleben & $1(2)$ \\
Ansprechrate & $1(2)$ \\
Ereignisfreies Überleben & $1(2)$ \\
Komplette Remission & $1(2)$ \\
Sehr gutes molekulares Ansprechen & $1(2)$ \\
Komplettes zytogenetisches Ansprechen & $1(2)$ \\
Erhebung zur Lebensqualität & $20(45)$ \\
Ja & $24(55)$ \\
Nein & \\
Vergleichstherapie & $17(39)$ \\
Wirkstoff vs. Placebo & $13(30)$ \\
Wirkstoff vs. Standardtherapie & $11(25)$ \\
Wirkstoff zusätzlich zur Standardtherapie & $2(5)$ \\
Wirkstoff vs. Best Supportive Care bzw. Beobachtung & $1(2)$ \\
Keine Vergleichstherapie & $29(66)$ \\
Patienten pro Studie & $15(34)$ \\
$<1000$ Patienten pro Studie & \\
\hline 1000 Patienten pro Studie & \\
\hline PAR Eupen Pre & \\
\hline &
\end{tabular}

EPAR = European Public Assessment Report; RCT = randomisierte kontrollierte Studie. ${ }^{a}$ Beinhaltet auch Standardtherapie + Placebo vs. Standardtherapie + zu prüfender Wirkstoff.

\section{Verbesserung in der Qualität von Zulassungsstudien zu neuen onkologischen Wirkstoffen - aber weiterhin auch viele Mängel}

Um die Qualität der Zulassungsstudien von aktuell in der Hämatologie und Onkologie zugelassenen Arzneimitteln zu charakterisieren, extrahierten wir Angaben zum Studiendesign aus den öffentlich zugänglichen Berichten (European Public Assessment Report, EPAR) der EMA (Tab. 1).

Zur Therapie hämatologischer Neoplasien und solider Tumore erschienen im Auswertungszeitraum (01.01.2009 bis 13.08.2012) EPAR zu 29 Wirkstoffen in 39 Indikationen. Es handelte sich überwiegend um Indikationserweiterungen bereits eingeführter Wirkstoffe. Für diese Zulassungen bzw. Zulassungserweiterungen wurden insgesamt 44 relevante (pivotal) klinische Studien vorgelegt, die zum Teil durch Ergebnisse weiterer klinischer Studien gestützt wurden. Unsere
Auswertung ergab, dass sich die Qualität der Zulassungsstudien in verschiedener Hinsicht verbessert hat, und bestätigte somit eine frühere Analyse der Arzneimittelkommission der deutschen Ärzteschaft (AkdÄ) [3]. So wurden meist die von der EMA und der FDA empfohlenen primären Endpunkte - Gesamtüberleben (overall survival (OS)) und progressionsfreies Überleben (progression-free survival (PFS)) verwendet und nur eine Studie wurde als Phase-II-Studie ohne Vergleichsarm durchgeführt. Bis vor wenigen Jahren häufig verwendete, als Beleg eines patientenrelevanten Nutzens jedoch nicht geeignete Surrogatendpunkte - beispielsweise Tumorprogress oder Ansprechrate [3, 6] - wurden nur noch selten untersucht (Tab. 1).

Allerdings fielen auch weiterhin Mängel in den Zulassungsstudien auf. So stützte sich beispielsweise die Zulassung meist $(90 \%)$ nur auf eine randomisierte kontrollierte Studie (RCT) der Phase III, die zudem überwiegend offen durchge- 
führt wurde. Trotz eindeutiger Empfehlung der Zulassungsbehörden wurden in der Mehrzahl der Zulassungsstudien keine Daten erhoben zu den für Patienten sehr relevanten, tumorbedingten Symptomen («Patient-Reported Outcomes», PRO) - ein neben OS und PFS als Nutzenbeleg akzeptierter Endpunkt für neue Arzneimittel - und zur Lebensqualität [2, $6,7]$. Außerdem wurden bei etwa zwei Drittel der Studien weniger als 1000 Patienten eingeschlossen. Deshalb werden seltene, mitunter schwerwiegende und potenziell tödlich verlaufende Nebenwirkungen in den Zulassungsstudien häufig nicht erkannt. Darüber hinaus können kurze Nachbeobachtungszeiten und der Einschluss von Patienten ohne bzw. mit wenigen Begleiterkrankungen ebenfalls dazu beitragen, dass patientenrelevante Nebenwirkungen in Zulassungsstudien nicht erkannt werden. Eine Untersuchung, in der Daten zu unerwünschten Ereignissen aus der Datenbank des Sponsors mit den Festlegungen im Studienprotokoll und den zugehörigen Publikationen verglichen wurden, zeigte erhebliche Widersprüche. Sie weisen darauf hin, dass Informationen zu Nebenwirkungen aus klinischen Studien verloren gehen [8]. Eine aktuelle Untersuchung von «zielgerichteten Wirkstoffen» zur Behandlung onkologischer Erkrankungen verdeutlicht die lückenhaften Kenntnisse zur Sicherheit dieser Arzneimittel zum Zeitpunkt der Zulassung [9]. Analysiert wurden 12 Wirkstoffe mit nach der Zulassung aktualisierten Fachinformationen und 36 Publikationen zu den entsprechenden Zulassungsstudien. Es zeigte sich, dass von den schweren Nebenwirkungen, die in aktualisierten Fachinformationen beschrieben wurden, 39\% nicht in den publizierten Zulassungsstudien und $49 \%$ nicht in den ursprünglichen Fachinformationen beschrieben worden waren. Bei 42\% $(n=5)$ der Arzneimittel wurde im Median 4,3 Jahre nach der Zulassung eine «Black Box»Warnung, die höchste Sicherheitswarnstufe der FDA, in die Fachinformation aufgenommen [9, 10].

Auf andere Mängel von Zulassungsstudien für onkologische Wirkstoffe wurde bereits früher hingewiesen. So werden Studien nach Zwischenanalysen, die zu diesem Zeitpunkt einen statistisch signifikanten, für den Patienten aber bisweilen nicht relevanten Vorteil zugunsten des neuen Wirkstoffs zeigen, häufiger vorzeitig abgebrochen. Dies kann zu einer Überschätzung der Wirksamkeit und zur unzureichenden Bewertung der Sicherheit des Arzneimittels führen. Daraus ergibt sich die Gefahr, dass ein neuer Wirkstoff mit nicht belegtem Nutzen und unklaren Risiken vorschnell in die Routineversorgung eingeführt wird [11]. Da zwischen Beginn und Abschluss klinischer Studien der Phase III oft 3-4 Jahre liegen, gelegentlich sogar längere Zeiträume, entspricht die gewählte Vergleichstherapie zum Zeitpunkt der Zulassung häufig nicht mehr dem therapeutischen Standard. Dies erschwert die Nutzenbewertung neuer Arzneimittel gegenüber der zweckmäßigen Vergleichstherapie, beispielswiese im Rahmen der nach Inkrafttreten des Gesetzes zur Neuordnung des Arzneimittelmarktes (AMNOG) vorgesehenen frühen Nutzenbewertung [12].
Ein weiteres Problem, der Publikationsbias, betrifft Zulassungsstudien insgesamt. Als Publikationsbias bezeichnet man die systematisch verzerrte Datenlage publizierter Studienergebnisse, die daraus resultiert, dass Zulassungsstudien nur unvollständig oder, bei negativen Ergebnissen, gelegentlich überhaupt nicht publiziert werden [13]. Eine wissenschaftlich fundierte Nutzenbewertung neuer Arzneimittel und Planung weiterer klinischer Studien nach Zulassung (Phase IV) zur Beantwortung offener, patientenrelevanter Fragen erfordern aber zwingend die Kenntnis und Bewertung aller Studienergebnisse. Der Publikationsbias, der auch in der Onkologie weiterhin $\mathrm{zu}$ finden ist [14-16], beeinflusst die Aussagen in systematischen Übersichtsarbeiten und Metaanalysen, aber auch evidenzbasierte klinische Entscheidungen sowie Leitlinien [13].

Um dem Publikationsbias entgegenzuwirken, müssen so schnell wie möglich umfassende rechtliche Verpflichtungen zur öffentlichen Registrierung von Studienprotokollen und -ergebnissen eingeführt werden. Ein Vergleich der in den USA und Europa der Öffentlichkeit zugänglichen Studienregister zu klinischen Studien für Arzneimittel verdeutlicht, dass die gesetzlichen Regelungen in den USA heute deutlich weiter gehen als in Europa [17, 18]. In den USA besteht eine umfassende Pflicht zur Registrierung aller Studien zu Arzneimitteln, Biopharmazeutika und Medizinprodukten sowie der Veröffentlichung einer Zusammenfassung der Ergebnisse klinischer Studien in einem jedermann zugänglichen Register [17]. Bei Nichtbefolgung dieser Vorgaben können spürbare Sanktionen (z.B. Geldstrafen von bis zu 10000 USD/Tag) verhängt werden. Demgegenüber existiert in der Europäischen Union (EU) bislang lediglich eine Registrierungspflicht klinischer Studien in der für die Öffentlichkeit derzeit nicht zugänglichen European Union Drug Regulating Authorities Clinical Trial(EudraCT)-Datenbank. Eine Pflicht zur Veröffentlichung von Studienergebnissen gibt es bislang nur für pädiatrische Studien, wobei die technischen Voraussetzungen hierfür jedoch noch nicht geschaffen sind. Eine Veröffentlichung der Zusammenfassung von Studienergebnissen auch bei Erwachsenen soll anlässlich der Fertigstellung einer neuen Version von EudraCT (V 9.0) Ende 2012 erfolgen [17, 18]. Inwieweit die im Rahmen des AMNOG verabschiedete Novelle des Arzneimittelgesetzes (AMG § 42b) - mit Verpflichtung zur Offenlegung von Ergebnissen klinischer Prüfungen - das Problem des Publikationsbias lösen wird, bleibt abzuwarten. Darüber hinaus wird der Status quo des begrenzten Zugangs zu klinischen Studienberichten (Clinical Study Reports), die heute nur den Zulassungsbehörden vorliegen, zu Recht infrage gestellt [19]. Öffentlich zugängliche, detaillierte Berichte - beispielsweise in Form eines Link im EPAR - sind unverzichtbar, um Wirksamkeit und Risiken neuer Arzneimittel in der Onkologie methodisch nicht verzerrt bewerten zu können [3]. 


\section{Zulassungsstudien für Arzneimittel zur Behandlung} von seltenen Leiden

Seltene Leiden betreffen definitionsgemäß weniger als 5 von 10000 Menschen in der EU bzw. weniger als 200000 Menschen in den USA. Weil pharmazeutische Unternehmen die Entwicklung von Arzneimitteln für seltene Leiden (Orphan Drugs (OD)) wegen hoher Kosten und geringer Umsatzerwartungen lange Zeit vernachlässigten, wurden gezielt Anreize geschaffen, um die Entwicklung dieser Arzneimittel zu fördern. Dazu gehören z.B. die wissenschaftliche Beratung bei der Erstellung des Prüfplans, der Erlass der Zulassungsgebühren und die Exklusivität der Vermarktungsrechte [20-23].

In der Hämatologie und Onkologie werden neue Arzneimittel zunehmend häufig als OD zugelassen - mehr als 50\% der in den letzten 10 Jahren von der EMA zugelassenen 64 OD betreffen hämatologische oder onkologische Indikationen [22] - und anschließend, auch aufgrund der eng definierten Anwendungsgebiete, häufig außerhalb der Zulassung (off label) eingesetzt [20-24].

Die Ergebnisse aus Studien für die Zulassung von OD sind vielfach weniger aussagekräftig als die aus Studien für die Zulassung von Wirkstoffen zur Behandlung nicht seltener onkologischer Krankheiten [23]. Dies veranschaulicht auch ein aktueller Vergleich der von der FDA für die Zulassung in der Onkologie herangezogenen klinischen Studien (Zeitraum der Auswertung: Januar 2004 bis Dezember 2010) für OD $(\mathrm{n}=15)$ versus NonOD $(\mathrm{n}=12)$ [20]. In die Zulassungsstudien für OD wurden deutlich weniger Patienten eingeschlossen (im Median 96 versus 290 Patienten bei Studien mit NonOD). Häufig wurde für die klinischen Studien zu OD ein offenes, nicht randomisiertes Design gewählt. Darüber hinaus wurden signifikant häufiger Surrogatendpunkte zur Bewertung der Wirksamkeit (z.B. Ansprechrate bzw. PFS) verwendet.

\section{Verbesserungsvorschläge: Was sollte sich vor und nach der Zulassung ändern?}

Eine Verbesserung der Datenlage zur Bewertung des Nutzens, aber auch der Kosten-Nutzen-Relation neu zugelassener Arzneimittel ist für eine rationale Verordnung kostenintensiver Wirkstoffe in der Onkologie unverzichtbar. Dafür müssen sowohl Standards für die präklinische Krebsforschung erhöht [25] als auch die heute (noch) gültigen Empfehlungen in der Verordnung (EG) Nr. 726/2004 strenger beachtet [2] und Mängel in den klinischen Studien vor der Zulassung weiter abgebaut werden [3]. Bei der Vielzahl der zu prüfenden neuen Arzneimittel und immer häufiger stratifizierenden Therapiestrategien ist die Entwicklung und Verwendung innovativer Studiendesigns notwendig [26, 27]. Dazu gehören beispielsweise adaptive Studien, in der nach ersten Zwischenauswertungen Anpassungen der Studie, z.B. hinsichtlich der
Fallzahl oder der zu prüfenden Dosisstufen, ermöglicht werden [26]. Gerade für Studien bei seltenen Erkrankungen sind alternative Studiendesigns und Endpunkte besonders relevant. Hier bietet sich die Zusammenarbeit der EMA mit kooperativen Studiengruppen an und eine mit der Zulassung verbundene Verpflichtung zur raschen Durchführung von Versorgungsstudien.

Für eine weitere Verbesserung der Qualität von RCT in der Onkologie sind zahlreiche Vorschläge entwickelt worden. Wesentliche Punkte sind:

- die Beachtung der CONSORT-Empfehlungen zum Studiendesign und zur Veröffentlichung von Studienergebnissen, einschließlich unerwünschter Ereignisse [28, 29];

- die verstärkte Förderung von Phase-III-Studien mit dem Potenzial, die ärztliche Versorgungspraxis $\mathrm{zu}$ verbessern [30];

- bei Verwendung von Surrogatendpunkten als Ersatz für den Goldstandard «Überlebenszeit» Erhebung auch von Daten zu PRO bzw. Lebensqualität [6, 7];

- Unterschiede in den Endpunkten zwischen dem Kontrollarm und dem experimentellen Arm, die nicht nur statistisch signifikant, sondern vor allem klinisch relevant sind [31];

- die Standardisierung der optimalen supportiven Therapie (best supportive care (BSC)) [32];

- die detaillierte Beschreibung der wesentlichen Einzelheiten zur Therapie, die für die Umsetzung der Studienergebnisse in der klinischen Praxis notwendig sind: beispielsweise die Prämedikation, der Einsatz von Wachstumsfaktoren oder Anpassungen der Dosis [33].

Nach Zulassung von neuen Arzneimitteln in der Onkologie müssen von ökonomischen Interessen und regulatorischen Vorgaben unabhängige klinische Studien (Post-Zulassungsstudien) rasch die noch offenen versorgungsrelevanten Fragen klären. Insbesondere gilt es, unter Alltagsbedingungen zu bestimmen: den relativen Nutzen (relative effectiveness) bzw. Zusatznutzen (added therapeutic value, d.h. bessere Wirksamkeit und/oder geringere Toxizität und/oder für den Patienten angenehmere Verabreichung) neu zugelassener Arzneimittel im Vergleich zu den verfügbaren Therapiealternativen. Dabei kann sich auch die Notwendigkeit ergeben, in einigen Kriterien von der Zulassung abzuweichen (z.B. Erweiterung des Patientenkollektivs durch veränderte Einschlusskriterien, neue Indikationen, andere Dosierung und/oder Dosierungsintervalle), um versorgungsrelevante Fragestellungen bearbeiten zu können. Diese unabhängigen klinischen Studien nach der Zulassung sind unverzichtbare Voraussetzung für einen bedarfsorientierten Einsatz medikamentöser onkologischer Therapien.

Die Sicherheit von Arzneimitteln nach der Zulassung soll durch verschiedene Maßnahmen überwacht werden. Dazu zählen Risikomanagementprogramme, das Spontanmeldesystem, laufende klinische Studien und Phase-IV-Studien nach der Zulassung [10]. 
Eine ausführliche Diskussion der Herausforderungen und Lösungsansätze für die Sicherstellung einer effizienten Arzneimittelversorgung in der Onkologie finden sich in einem Gutachten, das im Rahmen des Nationalen Krebsplans für das Bundesministerium für Gesundheit erstellt wurde, und im Arbeitspapier eines informellen Gesprächskreises [34, 35]. Behandelt werden in diesen Stellungnahmen auch weitere wichtige Aspekte zu Post-Zulassungsstudien, wie deren Aus- gestaltung, Verbesserung ihrer regulatorischen und finanziellen Rahmenbedingungen sowie Verminderung ihres administrativen Aufwands [34].

\section{Disclosure Statement}

Beide Autoren haben keine Interessenkonflikte.

\section{Literatur}

1 Mullard A: 2011 FDA drug approvals. Nat Rev Drug Discov 2012;11:91-94.

2 European Medicines Agency, Committee for Medicinal Products for Human Use: Guideline on the evaluation of anticancer medicinal products in man. Doc. Ref. No. CPMP/EWP/205/95/Rev.3/ Corr., London, 14. Dezember 2005. www.tga.gov. au/pdf/euguide/ewp020595enrev3.pdf.

3 Schott G, Gökbuget N, Pachl H, Ludwig W-D: Klinische Studien in der Onkologie - Defizite und Lösungsvorschläge. Z Evid Fortbild Qual Gesundhwes 2011:105:657-664.

4 Trotta F, Leufkens HG, Schellens JH, Laing R, Tafuri G: Evaluation of oncology drugs at the European Medicines Agency and US Food and Drug Administration: when differences have an impact on clinical practice. J Clin Oncol 2011;29:2266-2272.

5 Johnson JR, Ning YM, Farrell A, et al.: Accelerated approval of oncology products: the Food and Drug Administration experience. J Natl Cancer Inst 2011;103:636-644.

6 McKee AE, Farrell AT, Pazdur R, Woodcock J: The role of the U.S. Food and Drug Administration review process: clinical trial endpoints in oncology. Oncologist 2010;15(suppl 1):13-18.

7 Rock EP, Scott JA, Kennedy DL, et al.: Challenges to use of health-related quality of life for Food and Drug Administration approval of anticancer products. J Natl Cancer Inst Monogr 2007;27-30.

8 Scharf O, Colevas AD: Adverse event reporting in publications compared with sponsor database for cancer clinical trials. J Clin Oncol 2006;24:3933-3938.

-9 Seruga B, Sterling L, Wang L, Tannock IF: Reporting of serious adverse drug reactions of targeted anticancer agents in pivotal phase III clinical trials. J Clin Oncol 2011;29:174-185.

10 Berlin JA, Glasser SC, Ellenberg SS: Adverse event detection in drug development: recommendations and obligations beyond phase 3. Am J Public Health 2008;98:1366-1371.

11 Apolone G, Tafuri G, Trotta F, Garattini S: A new anti-cancer drug in the market: Good news for investors or for patients? Eur J Cancer 2008;44:17861788 .

12 Gemeinsamer Bundesausschuss: Die Nutzenbewertung von Arzneimitteln nach § 35a SGB V www.g-ba.de/institution/themenschwerpunkte/arzneimittel/nutzenbewertung35a/\#abschnitt-2.
3 Schott G, Pachl H, Ludwig W-D: Publikationsbias in Abhängigkeit von der Art der Finanzierung bei klinischen Studien. Z Evid Fortbild Qual Gesundhwes 2010;104:314-322.

14 Kho ME, Brouwers MC: Conference abstracts of a new oncology drug do not always lead to full publication: proceed with caution. J Clin Epidemiol 2009;62:752-758.

15 Tam VC, Tannock IF, Massey C, et al.: Compendium of unpublished phase III trials in oncology: characteristics and impact on clinical practice. J Clin Oncol 2011;29:3133-3139.

16 Hayes DF: Clinical trials: The silent minority - unpublished data on cancer care. Nat Rev Clin Oncol 2011;8:631-632.

17 Zarin DA, Tse T, Williams RJ, et al.: The ClinicalTrials.gov results database - update and key issues. N Engl J Med 2011;364:852-860.

18 Quack C: Gesetzliche Regelungen zur Datentransparenz - USA und Europa im Vergleich. Z Evid Fortbild Qual Gesundhwes 2011;105:183-188.

19 Doshi P, Jones M, Jefferson T: Rethinking credible evidence synthesis. BMJ 2012;344:d7898.

20 Kesselheim AS, Myers JA, Avorn J: Characteristics of clinical trials to support approval of orphan vs nonorphan drugs for cancer. JAMA 2011;305: 2320-2326.

21 Schwabe U, Paffrath D (Hrsg): ArzneiverordnungsReport 2011. Berlin, Heidelberg, Springer, 2011.

22 Committee for Orphan Medicinal Products and the European Medicines, Westermark K, Holm BB, Soderholm M, Llinares-Garcia J, Riviere F, et al.: European regulation on orphan medicinal products: 10 years of experience and future perspectives. Nat Rev Drug Discov 2011;10:341-349.

23 Windeler J, Koch K, Lange S, Ludwig W-D: Arzneimittelneuordnungsgesetz: $\mathrm{Zu}$ guter Letzt ist alles selten. Dtsch Arztebl 2010;107:A2032-A2034.

24 Janzen JWC, Ludwig WD: Off-Label-Therapie: aktuelle Probleme aus Sicht der Arzneimittelkommission der deutschen Ärzteschaft. Z Rheumatol 2012;71:108-110.

25 Begley CG, Ellis LM: Drug development: Raise standards for preclinical cancer research. Nature 2012;483:531-533.
6 Rubin EH, Gilliland DG: Drug development and clinical trials - the path to an approved cancer drug. Nat Rev Clin Oncol 2012;9:215-222.

27 Ludwig WD: Möglichkeiten und Grenzen der stratifizierenden Medizin am Beispiel von prädiktiven Biomarkern und «zielgerichteten» medikamentösen Therapien in der Onkologie. Z Evid Fortbild Qual Gesundhwes 2012;106:11-22.

28 Moher D, Jones A, Lepage L: Use of the CONSORT statement and quality of reports of randomized trials: a comparative before-and-after evaluation. JAMA 2001:285:1992-1995.

29 Ioannidis JP, Evans SJ, Gotzsche PC, O’Neill RT, Altman DG, Schulz K, et al.: Better reporting of harms in randomized trials: an extension of the CONSORT statement. Ann Intern Med 2004;141: 781-788.

30 Booth CM, Tannock I: Reflections on medical oncology: 25 years of clinical trials - where have we come and where are we going? J Clin Oncol 2008; 26:6-8.

31 Ocana A, Tannock IF: When are 'positive' clinical trials in oncology truly positive? J Natl Cancer Inst 2011:103:16-20.

32 Cherny NI, Abernethy AP, Strasser F, Sapir R, Currow D, Zafar SY: Improving the methodologic and ethical validity of best supportive care studies in oncology: lessons from a systematic review. J Clin Oncol 2009;27:5476-5486.

33 Duff JM, Leather H, Walden EO, LaPlant KD, George TJ Jr: Adequacy of published oncology randomized controlled trials to provide therapeutic details needed for clinical application. J Natl Cancer Inst 2010;102:702-705.

34 Bundesministerium für Gesundheit: Handlungsfelder Nationaler Krebsplan. www.bundesgesundheitsministerium.de/praevention/nationaler-krebsplan/handlungsfelder/handlungsfeld-3-sicherstellung-einer-effizienten-onkologischen-behandlung. html.

35 Gesprächskreis Versorgungsqualität in der Onkologie: Versorgungsqualität in der Onkologie - Herausforderungen und Lösungsansätze am Beispiel der Arzneimitteltherapie. www.krebsgesellschaft. de/download/Versorgungsqualitaet_in_der_Onkologie_2011.pdf. 